\title{
SUMMABILITY MATRICES THAT PRESERVE ASYMPTOTIC EQUIVALENCE FOR IDEAL CONVERGENCE
}

\author{
JEFF CONNOR AND HAFIZE GUMUS
}

\begin{abstract}
A characterization of summability matrices that preserve the asymptotic equivalence of two sequences is given, where asymptotic equivalence is defined with respect to an ideal $I$ of subsets of $\mathbb{N}$. Pobyvanets (1980) gave necessary and sufficient conditions for a nonnegative summability matrix $A$ to have the property that, for two nonnegative sequences $x$ and $y$ bounded away from 0 , the ratio $A x / A y$ tends to 1 whenever $x / y$ tends to 1 ; an analogous characterization is given where the convergence of $x / y$ is with respect to an ideal $I$ as well as a new proof of Pobyvanets' theorem. Similar extensions are given of theorems of Marouf and Li, in particular characterizations of summabilty matrices that map sequences $x$ and $y$ such that $x / y$ is $I$-convergent to 1 to sequences with the property that the ratio of the sums of their tails or the ratio of the supremum of their tails also tend to 1 with respect to an ideal $J$.
\end{abstract}

The main results of this paper are characterizations of summability matrices that preserve the asymptotic equivalence of two sequences, where the measures of asymptotic equivalence are defined with respect to an ideal of subsets of $\mathbb{N}$. The work builds on, and generalizes, similar theorems characterizing matrices that preserve asymptotic equivalence when defined using the standard definition of sequential convergence. The starting point for this paper is a 1980 theorem of Pobyvanets [16], which gives necessary and sufficient conditions for a nonnegative summability matrix $A$ to have the property that, for two nonnegative sequences $x$ and $y$ bounded away from 0 , one has that $A x / A y$ tends to 1 whenever $x / y$ tends to 1 . In this paper we establish this result (and others) where the convergence of $x / y$ is with respect

2010 Mathematics Subject Classification. Primary 40A35; Secondary 40G15.

Key words and phrases. Ideal convergence, statistical convergence, asymptotic equivalence, statistical limit superior.

This paper is in final form and no version of it will be submitted for publication elsewhere.

Copyright (c) 2016 by ANUBIH. 
to an ideal, which is weaker than ordinary sequential convergence. We also characterize nonnegative summability matrices that preserve convergence with respect to an ideal.

We continue our investigations by establishing generalizations of some results of Marouf [13] and Li [12]. They established results similar to Pobyvanets' by studying summability matrices that map sequences $x$ and $y$ such that $x / y$ tends to 1 to sequences with the property that the ratio of the sums of their tails or the ratio of the supremum of their tails also tends to 1. An analog of Pobyvanets' result, where ordinary convergence is replaced by statistical convergence, was established by Patterson in 2003 [15]. All of these results will be extended to the setting where convergence is replaced by convergence with respect to an ideal.

This paper has focuses on the characterization of the summability matrices that, in some sense, preserve asymptotic equivalence. It is worth noting, however, that there is another active area of research in the preservation of asymptotic equivalence that is not pursued in this paper. This area began with with a paper of Patterson and Savas [14], in which they established necessary and sufficient conditions for sequences to be simultaneously asymptotical equivalent with respect to statistical convergence, lacunary statistical convergence and lacunary strong summability. Their results have been extended in a variety of directions by a number of different authors.

\section{Pobyvanets' theorem And Related Results}

The first section of this paper describes the results that are generalized in this paper, which is followed by a brief introduction to convergence with respect to an ideal. We begin with a key definition and some notation. We let $c_{0}, c$, and $\ell_{\infty}$ denote the collections of real-valued sequences that, respectively, converge to 0 , converge to a limit, and are bounded. Given a sequence $x=\left(x_{n}\right)$, we let $\|x\|=\sup \left\{\left|x_{n}\right|: n \in \mathbb{N}\right\}$. We also make use of the following definition throughout this paper:

Definition 1.1. Let $x$ and $y$ be two real-valued sequences:

(1) $x$ and $y$ are said to be asymptotically equivalent if $\lim _{n} \frac{x_{n}}{y_{n}}=1$. In this case we write $x \sim y$.

(2) Let $\delta>0$. We say that the sequence $x=\left(x_{n}\right)$ is an element of $P_{\delta}$ if $x_{n}>\delta$ for all $n \in \mathbb{N}$.

Pobyvanets characterized the nonnegative summability matrices that preserve asymptotic equivalence for bounded sequences in $P_{\delta}([16])$. 
Theorem 1.2. (Pobyvanets, 1980) Let $T=\left(t_{n k}\right)$ be a nonnegative matrix. Then the following are equivalent:

(1) If $x, y \in P_{\delta} \cap l_{\infty}$ for some $\delta>0$ and $x \sim y$, then $T x \sim T y$.

(2) Given a finite subset $A \subset \mathbb{N}$,

$$
\lim _{n \rightarrow \infty} \frac{\sum_{k}\left\{t_{n k}: k \in A\right\}}{\sum_{k=1}^{\infty} t_{n k}}=0 .
$$

In [10], we offer an alternative proof for (2) implies (1) and extend Pobyvanets' result to broader setting; we will adopt a similar approach, and further extend the result in that paper. The rough idea of the approach is, given a matrix $T$ that we wish to show preserves asymptotic equivalence, we use the hypothesis on $T$ to create a regular matrix $S$ and then exploit the regularity of $S$ to show that $T$ has the desired property. A key observation, which is generalized in Remark 2.4, is that if $x, y \in P_{\delta} \cap l$, then $x \sim y$ if and only if $\lim (x-y)=0$.

It is worth noting, as the next two examples demonstrate, that the assumptions that $T \geq 0$ and that $y \in P_{\delta}$ cannot be easily removed from the hypothesis of the theorem.

First we observe that there is a matrix where $T \nsupseteq 0$ and the conclusion of Pobyvanets' theorem is false. Let $\alpha>0$ and define $T$ by

$$
T=\left[\begin{array}{cccccccc}
1+\alpha & -\alpha & 0 & 0 & 0 & 0 & 0 & \ldots \\
0 & 0 & 1+\alpha & -\alpha & 0 & 0 & 0 & \ldots \\
0 & 0 & 0 & 0 & 1+\alpha & -\alpha & 0 & \ldots \\
\vdots & \vdots & \vdots & \vdots & \vdots & \vdots & \vdots &
\end{array}\right]
$$

Note that $T$ satisfies condition 2) of Pobyvanets' theorem. Now set

$$
z=1, \frac{1+\alpha}{\alpha}, 1, \frac{1+\alpha}{\alpha}, 1, \frac{1+\alpha}{\alpha}, \ldots
$$

and observe that $(T z)_{n}=0$ for all $n$.

Now let $\delta=\left(\delta_{n}\right)$ be a positive-valued sequence decreasing to zero. Set $x=z+\delta$ and $y=z-\delta$ and note $x \sim y$. Yet

$$
\lim _{n} \frac{(T x)_{n}}{(T y)_{n}}=\lim _{n} \frac{(T z)_{n}+(T \delta)_{n}}{(T z)_{n}-(T \delta)_{n}}=\lim _{n} \frac{(T \delta)_{n}}{-(T \delta)_{n}}=-1 .
$$

Hence $x \sim y$ but $T x$ is not asymptotically equivalent to $T y$.

Next we observe that, even if $T \geq 0$, then the condition that $y \in P_{\delta}$ cannot not be omitted. Let $(T x)_{n}=\frac{\bar{x}_{1}}{2^{n}}+x_{n}$ for $n>1$. Then $T$ satisfies the condition

$$
\lim _{n \rightarrow \infty} \frac{\sum_{k}\left\{t_{n k}: k \in A\right\}}{\sum_{k=1}^{\infty} t_{n k}}=0
$$


for every finite subset $A \subset \mathbb{N}$. Now set

$$
x=2,2^{-2}, \ldots, 2^{-2 n}, \ldots \text { and } y=1,2^{-2}, \ldots, 2^{-2 n}, \ldots
$$

Note $x, y \rightarrow 0$ and $x \sim y$, yet

$$
\lim _{n} \frac{(T x)_{n}}{(T y)_{n}}=\lim _{n} \frac{2+2^{-n}}{1+2^{-n}}=2
$$

and hence $T x \nsim T y$.

J. Fridy, in a study of the comparison of the rates of convergence of series, introduced two other techniques for comparing rates of convergence [6] that have been used to study the asymptotic equivalence of sequences. One, which we shall denote as $R$-asymptotic equivalence, considers the ratio of the sums of the tails of the series. The other, which we will call $\mu$-equivalence, considers the ratio of the supremum of the tails of a sequence; this test, in its original form, was used to compare how fast two series converged to their respective sums. Following Marouf, we use the following definition:

Definition 1.3. Let $x$ and $y$ be two real valued sequences.

(1) Let $w$ be a sequence and set $R_{n}(w)=\sum_{k \geq n}\left|w_{k}\right|$. Then $x$ and $y$ are $R$ asymptotically equivalent provided that $\lim _{n}\left(R_{n}(x) / R_{n}(y)\right)=1$; in this case we write $R x \sim R y$.

(2) Let $w$ be a sequence and set $\mu_{n}(w)=\sup _{k \geq n}\left\{w_{k}: k \geq n\right\}$. Then $x$ and $y$ are $\mu$-asymptotically equivalent provided that $\lim _{n}\left(\frac{\mu_{n}(x)}{\mu_{n}(y)}\right)=1$; in this case we write $\mu x \sim \mu y$.

Pobyvanets' theorem served as the starting point for a paper of Marouf [13], which was followed by a paper of $\mathrm{Li}$ [12], and we will continue their investigations in the setting of convergence with respect to an ideal. Marouf examined matrices for which $x \sim y$ implies $R x \sim R y$. In the course of his investigations he established the following result:

Theorem 1.4. (Marouf, 1993) Let $T \geq 0$ and $T: l_{\infty} \rightarrow l_{1}$. Then the following are equivalent:

(1) If $x, y \in P_{\delta} \cap l_{\infty}$ and $x \sim y$, then $R x \sim R y$.

(2) For every finite subset $A \subset \mathbb{N}$

$$
\lim \frac{\sum_{j=n}^{\infty} \sum_{k}\left\{t_{j k}: k \in A\right\}}{\sum_{j=n}^{\infty} \sum_{k=1}^{\infty} t_{j k}}=0 .
$$

Marouf's proof of this result used calculations based on the definition of $R_{n}$ : the delicate part of these computations involved using the assumption that the terms of $y$ are bounded away from 0 by $\delta$ and that $x$ is bounded to show that $R_{n}(T x) / R_{n}(T y)<1+\varepsilon$ (for any $\varepsilon>0$ ) when $x \sim y$. In 
this paper we will extend this result using a different proof that is similar to the proof given for Pobyvanets' theorem. Marouf also showed that if $T$ is a nonnegative matrix that maps $c_{0}$ into $c_{0}, x, y \in P_{\delta} \cap l_{\infty}$, and $x \sim y$, then $\mu(T x) \sim \mu(T y)$. In a later paper, Li [12] characterized nonnegative matrices which preserve $\mu$-equivalence. This result can also be extended to the setting of convergence with respect to an ideal, as will be demonstrated in the last section of this paper.

\section{A short introduction to CONVERGEnCE With Respect to AN IDEAL}

Before moving to the main results of this paper, however, we pause for a brief introduction to sequential convergence with respect to an ideal. Sequential convergence with respect to an ideal was introduced by Kostryko, Salát, and Wilczyński [11], and is a generalization of statistical convergence as introduced by Fast [5] and developed by Fridy with respect to summability theory in his frequently cited paper On Statistical Convergence [7]. Our paper only uses the elementary aspects of this theory and we will include any necessary details as they are needed.

Definition 2.1. A collection $I$ of subsets of $\mathbb{N}$ is called an admissible ideal provided 1) $\phi \in I$; 2) $A, B \in I$ implies $A \cup B \in I$; and 3) $B \in I$ and $A \subset B$ implies $A \in I$. If the ideal $I$ contains the finite subsets of $\mathbb{N}$ and $\mathbb{N} \notin I$, then $I$ is called an admissible ideal.

A rudimentary example of an admissible ideal is the set of all finite subsets of $\mathbb{N}$. As has been noted by others, many of the traditional objects of study in summability theory, such as regular summability matrices, can be used to generate ideals. A well-known ideal is collection of subset of $\mathbb{N}$ with natural density zero, i.e.,

$$
\left\{A \subset \mathbb{N}: \lim _{n} \frac{1}{n} \sum_{k \leq n} \chi_{A}(k)=0\right\}
$$

This corresponds with the sequences of $0^{\prime} s$ and $1^{\prime} s$ that are statistically convergent to 0 and is the ideal generated by the Cesaro summability matrix. There is also a strong connection between ideals of $\mathbb{N}$ and two-valued measures defined on subalgebras of subsets of $\mathbb{N}$.(cf. [2]).

The definition of convergence with respect to an ideal has it roots in observing that a sequence $\left(x_{n}\right)$ is convergent to $L$ if and only if, for every $\varepsilon>0$, the set $\left\{n:\left|x_{n}-L\right| \geq \varepsilon\right\}$ is in the ideal of finite subsets of $\mathbb{N}$. This in mind, we recall the following definition ([11]).

Definition 2.2. Let $I$ be an ideal of subsets of $\mathbb{N}, x=\left(x_{n}\right)$ be a (realvalued) sequence and $L \in \mathbb{R}$. The sequence $x$ is said to be $I$-convergent to 
$L$, and we write $I-\lim x=L$, provided

$$
\left\{n:\left|x_{n}-L\right| \geq \varepsilon\right\} \in I
$$

for every $\varepsilon>0$. We let $c_{I}$ denote the I-convergent sequences.

It is well known, and routine to establish, that the $I$-limit is a linear functional as well as satisfying other properties of the limit function. Ordinary convergence is preserved by $I$-convergence for admissible ideals, i.e., if $I$ is admissible and $x$ is a convergent sequence, then $I-\lim x$ exists and equals $\lim x$. That said, some caution needs to be used in working with the $I$-limit. For instance, $I$-convergent sequences are not necessarily bounded and the $I$-limit is not necessarily translation invariant.

We are now ready to extend the definition of asymptotic equivalence to the setting of convergence with respect to an ideal.

Definition 2.3. We say that the sequences $x$ and $y$ are $I$-asymptotically equivalent if $I-\lim (x / y)=1$; in this case we write $x \sim_{I} y$.

For sequences $x$ and $y$, we let $R x \sim_{I} R y$ and $\mu x \sim_{I} \mu y$ denote, respectively, the $I$-asymptotic equivalence of $R x$ and $R y$ and the $I$-asymptotic equivalence of $\mu x$ and $\mu y$ (see Definition 1.3 for the definition of $R x$ and $\mu x)$.

Given an ideal $I$, it will often be convenient to work with the filter associated with $I$, which is denoted $F(I)$ and defined by $F(I)=\left\{A \subseteq \mathbb{N}: A^{c} \in I\right\}$. Note that $F(I)$ satisfies the defining properties of a filter, i.e., 1) $\phi \notin F(I)$, 2) $F(I)$ is closed under finite intersection, and 3) if $A \in F(I)$ and $A \subseteq B$, then $B \in F(I)$. It is often convenient to think of the members of $I$ as having ' $I$-density 0 ' and the members of $F(I)$ as having ' $I$-density 1 '. The property of being admissible ideal corresponds to the associated filter containing no finite sets.

The following remark, given in [10], will be useful in the remainder of the paper.

Remark 2.4. Let $I$ be an admissible ideal and let $y$ be a bounded sequence.

(1) If $y$ is a bounded sequence, $\left\{k: y_{k}>0\right\} \in F(I)$, and $x$ is a sequence such that $I-\lim (x / y)=1$, then $I-\lim (x-y)=0$.

(2) If there is a $\delta>0$ such that $\left\{k: y_{k}>\delta\right\} \in F(I)$ and $x$ is a sequence such that $I-\lim (x-y)=0$, then $I-\lim (x / y)=1$.

Proof. 1.) Let $\varepsilon>0$ be given. Since $I-\lim (x / y)=1$, we have that

$$
A=\left\{n:\left|\frac{x_{n}}{y_{n}}-1\right|<\frac{\varepsilon}{2\|y\|}\right\} \in F(I)
$$


and, if $n \in A$, then

$$
\left|x_{n}-y_{n}\right|<\left(\frac{y_{n}}{2\|y\|}\right) \varepsilon<\varepsilon .
$$

Hence $A \subset\left\{n:\left|x_{n}-y_{n}\right|<\varepsilon\right\}=B$ and, as $B \in F(I)$ and $\varepsilon$ was arbitrary, $I-\lim (x-y)=0$.

2.) Let $\varepsilon>0$. Note that if $\left|x_{n}-y_{n}\right|<\varepsilon \delta$ and $y_{n}>\delta$, then

$$
\left|\frac{x_{n}}{y_{n}}-1\right|<\frac{\varepsilon \delta}{y_{n}}<\varepsilon
$$

Hence

$$
A=\left\{n:\left|x_{n}-y_{n}\right|<\varepsilon \delta\right\} \subset\left\{n:\left|\frac{x_{n}}{y_{n}}-1\right|<\varepsilon\right\}=B
$$

and, as $A \in F(I)$, it follows that $B \in F(I)$. Since $\varepsilon$ was arbitrary, $I-$ $\lim (x / y)=1$.

\section{Ideals \& Pobyvanets theorem}

Now we turn our attention to extending Pobyvanets' result to the setting of ideal convergence. The result closely parallels the statement of Pobyvanets' theorem and its proof follows the one described in the introduction. The first step is to establish a result similar to the Silverman-Toeplitz theorem for the special case of nonnegative matrices that map the bounded sequences into themselves, which similar to a more general result given in [1] and extends a theorem stated (without proof) in [10].

Theorem 3.1. Let $I$ and $J$ be admissible ideals and suppose that $T$ is a nonnegative matrix which maps bounded sequences to bounded sequences. Then the following are equivalent:

(1) $I-\lim x=J-\lim T x$ for all $x \in c_{I} \cap l_{\infty}$.

(2) $A \in I$ implies $J-\lim \sum_{k}\left\{t_{n k}: k \notin A\right\}=1$.

Proof. First suppose that $I-\lim x=J-\lim T x$ for all $x \in c_{I} \cap l_{\infty}$. If $A \in I$, then we have that, $\chi_{A^{c}}$ is $I$-convergent to 1 and hence, by assumption,

$$
J-\lim T\left(\chi_{A^{c}}\right)=J-\lim \sum_{k}\left\{t_{n k}: k \notin A\right\}=1 .
$$

Hence (1) implies (2).

Next we establish that (2) implies (1). Before starting we make two observations. First note that if $A \in I$, then $J-\lim T\left(\chi_{A^{c}}\right)=J-\lim T\left(\chi_{\mathbb{N}}\right)=1$, thus

$$
J-\lim \sum_{k \in A} t_{j k}=J-\lim T\left(\chi_{\mathbb{N}}\right)-\left(J-\lim T\left(\chi_{A^{c}}\right)\right)=0
$$


and we have that $\left\{j:\left|\sum_{k \in A} t_{j k}\right| \geq \varepsilon\right\} \in J$ for all $\varepsilon>0$. Second, if $x \in$ $c_{I} \cap l_{\infty}$, then $I-\lim x=L$ if and only if $I-\lim (x-L)=0$, and hence it suffices to show that, for a bounded sequence $x$, condition (2) yields that $I-\lim x=0$ implies $J-\lim T x=0$.

Having made these observations, we now establish that (2) implies (1). Let $x$ be a bounded sequence such that $I-\lim x=0$ and let $\varepsilon>0$ be given. Set $A=\left\{k:\left|x_{k}\right| \geq \frac{\varepsilon}{3}\right\}$ and note that $A^{c} \in F(I)$. Define

$$
B_{1}=\left\{j:\left|\sum_{k \in A^{c}} t_{j k}-1\right|<\frac{1}{2}\right\} \text { and } B_{2}=\left\{j:\left|\sum_{k \in A} t_{j k}\right|<\frac{\varepsilon}{2\|x\|}\right\} .
$$

Observe that (2) yields that $B_{1} \in F(J)$, that $J-\lim T\left(\chi_{A}\right)=0$ yields that $B_{2} \in F(J)$, and consequently $B_{1} \cap B_{2} \in F(J)$. Let $j \in B_{1} \cap B_{2}$ and note that

$$
\begin{aligned}
\left|\sum_{k} t_{j k} x_{k}\right| & \leq\left|\sum_{k \in A^{c}} t_{j k} x_{k}\right|+\left|\sum_{k \in A} t_{j k} x_{k}\right| \\
& \leq \frac{\varepsilon}{3} \sum_{k \in A^{c}} t_{j k}+\|x\| \sum_{k \in A} t_{j k} \\
& <\frac{\varepsilon}{3} \cdot \frac{3}{2}+\|x\| \cdot \frac{\varepsilon}{2\|x\|}=\varepsilon .
\end{aligned}
$$

It follows that $B_{1} \cap B_{2} \subset\left\{j:\left|\sum t_{j k} x_{k}\right|<\varepsilon\right\}$, and hence $\left\{j:\left|\sum t_{j k} x_{k}\right|<\varepsilon\right\}$ $\in F(J)$. As $\varepsilon$ was arbitrary, $J-\lim (T x)=0$.

Lemma 3.2. Let $S$ be a nonnegative matrix that $S$ maps bounded I-convergent sequences to bounded $J$-convergent sequences with the property that $I-\lim x=J-\lim S x$ for every $x \in c_{I} \cap \ell_{\infty}$. If $x, y \in P_{\delta} \cap \ell_{\infty}$ and $x$ is I-asymptotically equivalent to $y$, then $S x$ is $J$-asymptotically equivalent to Sy.

Proof. Observe that the complement of $A=\left\{k: x_{k}>\delta\right\}$ is finite and hence $J-\lim S\left(\chi_{A}\right)=I-\lim \chi_{A}=1$, and it follows that $B=\left\{j: \sum_{l} s_{j l} \chi_{A}(l)>\frac{1}{2}\right\}$ $\in F(J)$. Hence, for $j \in B$,

$$
\sum_{l} s_{j l} x_{j} \geq \sum_{l \in A} s_{j l} x_{l}>\delta \sum_{l \in A} s_{j l}>\frac{\delta}{2}
$$

and consequently $x, y \in P_{\delta} \cap \ell_{\infty}$ yields that $S x, S y \in P_{\frac{\delta}{2}} \cap \ell_{\infty}$. Now

$$
\begin{aligned}
& x \sim{ }_{I} y \Rightarrow I-\lim (x-y)=0 \\
& \quad \Rightarrow J-\lim (S x-S y)=0 \Rightarrow S x \sim_{J} S y .
\end{aligned}
$$


We now offer the generalization of Pobyvanets' theorem.

Theorem 3.3. Let $I$ and $J$ be and admissible ideals, $T$ be a nonnegative matrix and suppose that $x$ and $y$ are bounded elements of $P_{\delta}$. Then the following are equivalent:

(1) $x \sim_{I} y$ implies $T x \sim_{J} T y$

(2) For every $A \in I$,

$$
J-\lim \frac{\sum_{k}\left\{t_{j k}: k \in A\right\}}{\sum_{k=1}^{\infty} t_{j k}}=0 .
$$

Proof. First we establish that (2) implies (1). Let $S=\left(s_{j k}\right)$ be defined by

$$
s_{j k}=\frac{t_{j k}}{\sum_{k} t_{j k}} .
$$

Note that if $A \in I$, then

$$
J-\lim \sum_{j} s_{j k} \chi_{A}(k)=J-\lim \frac{\sum_{k}\left\{t_{j k}: k \in A\right\}}{\sum_{k=1}^{\infty} t_{j k}}=0
$$

and hence, by the preceding lemma $S x \sim_{J} S y$ whenever $x, y \in P_{\delta} \cap \ell_{\infty}$ and $x \sim_{I} y$. Now

$$
J-\lim _{n} \frac{(T x)_{n}}{(T y)_{n}}=J-\lim _{n} \frac{\frac{(T x)_{n}}{\sum_{k=1}^{\infty} t_{n k}}}{\frac{(T y)_{n}}{\sum_{k=1}^{\infty} t_{n k}}}=J-\lim _{n} \frac{(S x)_{n}}{(S y)_{n}}=1,
$$

i.e., $T x$ is $J$-asymptotically equivalent to $T y$.

The proof that (1) implies (2) is as follows. Suppose there is an $A \in I$ such that

$$
J-\lim \frac{\Sigma_{k} t_{j k} \chi_{A}(k)}{\Sigma_{k} t_{j k}} \neq 0 .
$$

Then there is an $\nu>0$ and a $B \notin J$ such that for every $j \in B$

$$
\frac{\Sigma_{k} t_{j k} \chi_{A}(k)}{\Sigma_{k} t_{j k}}>\nu>0 .
$$

Define the sequence $x=\left(x_{k}\right)$ by $x_{k}=2$ for $k \in A$ and $x_{k}=1$ for $k \notin A$ and let the sequence $y=\left(y_{k}\right)$ be defined by $y_{k}=1$ for all $k \in \mathbb{N}$. Note that $I-\lim (x / y)=1$ and both are bounded members of $P_{1 / 2}$. Now, if $j \in B$, then

$$
\frac{\Sigma t_{j k} x_{k}}{\Sigma t_{j k} y_{k}}=\frac{\Sigma_{k \in A} t_{j k}+\Sigma t_{j k} x_{k}}{\Sigma t_{j k}}>\nu+1>1
$$

and hence $T x$ is not $J$-asymptotically equivalent to $T y$. 


\section{IDEAls \& $R$-EQUivalence}

We next turn to establishing an $I-J$ version of Marouf's theorem for $R$-asymptotic equivalence. The following proof is based on a factorization scheme that allows us to apply the $I-J$ version of Pobyvanets' theorem.

Theorem 4.1. Let $T \geq 0$ and $T: l_{\infty} \rightarrow l_{1}$. Then the following are equivalent:

(1) If $x, y \in P_{\delta} \cap \ell_{\infty}$ and $x \sim_{I} y$, then $R x \sim_{J} R y$.

(2) For every $A \in I$

$$
J-\lim _{n} \frac{\sum_{j=n}^{\infty} \sum_{k}\left\{t_{j k}: k \in A\right\}}{\sum_{j=n}^{\infty} \sum_{k} t_{j k}}=0 .
$$

Proof. The proof proceeds by using $T$ to define a matrix $C$ with the property that $C x$ is $J$-asymptotically equivalent to $C y$ if and only if

$$
J-\lim \frac{R_{n}(T x)}{R_{n}(T y)}=1 .
$$

Once $C$ has been defined, applying Theorem 3.3 to $C$ yields the promised equivalence for $T$.

Define the matrix $C=\left(c_{l k}\right)$ by $c_{l k}=\sum_{j=l}^{\infty} t_{j k}$. Note that, since $T: l_{\infty} \rightarrow$ $l_{1}$, each entry of $C$ is defined as well as, for each $l \in \mathbb{N}$, the sum $\sum_{j=l}^{\infty} \sum_{k} t_{j k}$. Observe that for $l \in \mathbb{N}$ and $x \in \ell_{\infty}$ we have that

$$
\begin{aligned}
\sum_{k=1}^{\infty} c_{l k} x_{k} & =\sum_{k=1}^{\infty} \sum_{j \geq l} t_{j k} x_{k}=\sum_{j \geq l}\left(\sum_{k=1}^{\infty} t_{j k} x_{k}\right) \\
& =R_{l}(T x)
\end{aligned}
$$

and hence $J-\lim \frac{R_{l}(T x)}{R_{l}(T y)}=1$ if and only $C x \sim_{J} C y$.

Now, note that

$$
\sum_{k=1}^{\infty} c_{l k} \chi_{A}(k)=\sum_{j \geq l}\left(\sum_{k=1}^{\infty} t_{j k} \chi_{A}(k)\right)
$$

and hence $C$ is $I-J$ asymptotically regular if and only if

$$
J-\lim \frac{\sum_{j=l}^{\infty} \sum_{k}\left\{t_{j k}: k \in A\right\}}{\sum_{j=l}^{\infty} \sum_{k} t_{j k}}=0
$$

for every $A \in I$.

The preceding argument contains a useful way of visualizing the proof of Theorem 4.1. Note that if we set $R$ to be the matrix which maps an absolutely convergent sequence $x$ to $\left(R_{l}(x)\right)$, then $C=R T$, i.e., we defined $C$ by 


$$
C=\left[\begin{array}{ccccc}
1 & 1 & 1 & 1 & \ldots \\
0 & 1 & 1 & 1 & \ldots \\
0 & 0 & 1 & 1 & \ldots \\
\vdots & \vdots & \vdots & \vdots &
\end{array}\right]\left[\begin{array}{ccccc}
t_{11} & t_{12} & t_{13} & t_{14} & \ldots \\
t_{21} & t_{22} & t_{23} & t_{24} & \ldots \\
t_{31} & t_{32} & t_{33} & \ldots & \ldots \\
\vdots & \vdots & \vdots & \vdots &
\end{array}\right]
$$

The manipulations in the preceding proof show that $(R T x)_{l}=R_{l}(T x)$. Theorem 4.1 is then established by applying Theorem 3.3 to $R T$. Condition (2) of Theorem 3.3 shows that $R T$ is $I-J$ asymptotically regular, which in turn yields condition (1) of Theorem 4.1. For the converse, condition (1) of Theorem 4.1 implies that $R T$ is $I-J$ asymptotically regular and, by using condition (2) of Theorem 3.3 and unwinding the matrix multiplication used to create $R T$, we obtain statement (2) of theorem 4.1.

One can also establish the result following Marouf's proof, which is more computationally intensive. One insight of using this approach is that the significance of the restrictions on $x$ and $y$ as well as the assumption $T \geq 0$ are more transparent. As the calculation proceeds, one arrives at the inequality:

$$
\begin{aligned}
\frac{R_{n}(T x)}{R_{n}(T y)} & \leq \frac{(1+\varepsilon / 2) \sum_{j=n}^{\infty} \sum_{k}\left\{t_{j k} y_{k}: k \in A\right\}}{\sum_{j=n}^{\infty} \sum_{k=1}^{\infty} t_{j k} y_{k}} \\
& \vdots \\
& +\frac{\|x\|}{\delta} \frac{\sum_{j=n}^{\infty} \sum_{k}\left\{t_{j k}: k \notin A\right\}}{\sum_{j=n}^{\infty} \sum_{k=1}^{\infty} t_{j k} y_{k}}
\end{aligned}
$$

where $A=\left\{k:\left|x_{k} / y_{k}-1\right|<\varepsilon / 2\right\}$. Note that $y \in P_{\delta}, x \in l_{\infty}$ and $T \geq 0$ all appear to be important to establishing this inequality.

\section{5. $\mu$-ASYMPTOTICALLY EQUIVALENT SEQUENCES}

In this section we generalize Li's result. Before moving to the generalization, though, it will necessary to reformulate the statement of the theorem. The preceding results suggest that one the natural $I-J$ extension of $\mathrm{Li}$ and Marouf's work would be to characterize matrix maps $T$ with the property that such that

$$
I-\lim \frac{\mu x}{\mu y}=1 \Longrightarrow J-\lim \frac{\mu T x}{\mu T y}
$$

Curiously, as the following observations show, this would just take us back to Li's result.

Marouf showed that if $T$ is a nonnegative matrix that maps $c_{0}$ into $c_{0}$, $x, y \in P_{\delta} \cap l_{\infty}$, and $x \sim y$, then $\mu(T x) \sim \mu(T y)$; the identity matrix $E$ shows that Marouf's result does not extend to $I$-convergence in general. In particular, if $I$ is an ideal that contains an infinite set, then $x \sim_{I} y$ does not 
imply $\mu(E x) \sim_{I} \mu(E y)$ since there are sequences $x, y \in P_{\delta} \cap l_{\infty}$ such that $I-\lim (x / y)=1$ and $I-\lim (\mu x / \mu y)=2$. To construct $x$ and $y$, suppose that $A \in I$ is an infinite set and define $x=\left(x_{k}\right)$ by $x_{k}=2$ for $k \in A$ and $x_{k}=1$ otherwise, and define $y=\left(y_{k}\right)$ by $y_{k}=1$ for all $k \in \mathbb{N}$. Now $x \sim_{I} y$ but, as $|A|=\infty$, we have that $\sup _{k \geq n} x_{k}=2$ for all $n \in \mathbb{N}$, and consequently $I-\lim (\mu x / \mu y)=2$.

We also observe that if $x, y \in P_{\delta} \cap l_{\infty}$, then

$$
I-\lim \frac{\mu x}{\mu y}=1 \Leftrightarrow \lim \frac{\mu x}{\mu y}=1 .
$$

and hence the matrices with the property that

$$
I-\lim \frac{\mu x}{\mu y}=1 \Longrightarrow J-\lim \frac{\mu(T x)}{\mu(T y)}=1
$$

are precisely the ones that $\mathrm{Li}$ characterized. To establish the observation, first note that, since ordinary convergence implies and is consistent with $I$ convergence, we have that $\lim \frac{\mu x}{\mu y}=1$ implies $I-\lim \frac{\mu x}{\mu y}=1$, i.e., $\mu x \sim_{I} \mu y$. The reverse implication follows from noting that $\lim _{n}\left(\sup _{k \geq n} x_{k}\right)=\lim \sup x$ and $\lim \sup y$ both exist and are greater than $\delta$, and hence $\lim (\mu x / \mu y)=$ $\beta$ for some $\beta$ and thus $I-\lim (\mu x / \mu y)=\beta$. As we have assumed $I-$ $\lim (\mu x / \mu y)=1$ it follows that $\lim \frac{\mu x}{\mu y}=1$, i.e., $\mu x \sim \mu y$. We also note that, if the hypothesis that $x, y \in P_{\delta} \cap l_{\infty}$ is dropped, then the preceding observation is no longer valid. In particular, if we remove the restriction that the sequences be bounded away from zero, then there are positive sequences $x$ and $y$, not bounded away from 0 from below, and an ideal $I$ such that $I-\lim \frac{\mu x}{\mu y}=1$ and $\lim \frac{\mu x}{\mu y}$ does not exist.

As the preceding shows, a meaningful extension of Li's result will have to entail some modification of the statement of the theorem. One can show that if $x, y \in P_{\delta} \cap l_{\infty}$, then

$$
\lim _{n} \frac{\mu x}{\mu y}=1 \Leftrightarrow \lim \sup \mu x=\lim \sup \mu y .
$$

Hence, given that $x, y \in P_{\delta} \cap l_{\infty}$, we could replace the hypothesis $\lim \frac{\mu x}{\mu y}=1$ with $\lim \sup \mu x=\lim \sup \mu y$ in Li's theorem. This suggests that a theorem characterizing nonnegative matrices with the property that

$$
I-\lim \sup x=I-\lim \sup y \Rightarrow J-\lim \frac{\mu T x}{\mu T y}=1
$$

would be an extension of Li's result once " $I-\lim \sup x$ " has been defined.

Fortunately, there is a commonly accepted definition of " $I-\lim$ sup" available to us. J. Fridy and C. Orhan first introduced the notion of a 
'statistical limit superior' in the context of statistical convergence [9]. This notion was extended to statistical convergence with respect to a density in [3] and to statistical convergence with respect to an ideal by Demirci [4], whose definition we use in this paper.

Definition 5.1. Let $x$ be a sequence and set

$$
B_{x}=\sup \left\{b \in \mathbb{R}:\left\{k: x_{k}>b\right\} \notin I\right\} .
$$

Then

$$
I-\lim \sup x=\left\{\begin{array}{cc}
\sup B_{x} & B_{x} \neq \phi \\
-\infty & B_{x}=\phi
\end{array}\right.
$$

The $I$ - limsup behaves in much the same way as the usual limsup. As one might expect, it is the case that $I-\lim x=l$ if and only if $I-\lim \sup x=$ $I-\liminf x=l$. for bounded sequences [4]. Some care, however, needs to taken: for instance, statistical cluster points are not identical to statistical limit points, as is the case for ordinary convergence [8]. We make use of the following theorem [4].

Theorem 5.2. If $\beta=I-\lim \sup x$ is finite, then for every positive number $\varepsilon$

$$
\left\{k: x_{k}>\beta-\varepsilon\right\} \notin I \text { and }\left\{k: x_{k}>\beta+\varepsilon\right\} \in I \text {. }
$$

Conversely, if the preceding relationship holds for every $\varepsilon>0$, then $I-$ $\lim \sup x=\beta$.

Note that if $I$ is the ideal finite sets, the above agrees with the description of the classical limit superior. The proof of the following theorem is similar in structure to Li's proof of his result [12].

Theorem 5.3. Let $I$ and $J$ be admissible ideals. If $T \geq 0, T: l_{\infty} \rightarrow l_{\infty}$, and $x, y \in P_{\delta} \cap l_{\infty}$, then the following are equivalent:

(1) If $I-\lim \sup x=I-\lim \sup y$,then

$$
J-\lim \frac{\mu T x}{\mu T y}=1 .
$$

(2) The following conditions are satisfied

(a) if $A \notin I$, then

$$
J-\lim \frac{\sup _{k \geq n}\left\{\sum_{l} t_{k l}: l \in A\right\}}{\sup _{k \geq n} \sum_{l} t_{k l}}=1
$$

(b) if $A \in I$, then

$$
J-\lim \frac{\sup _{k \geq n}\left\{\sum_{l} t_{k l}: l \in A\right\}}{\sup _{k \geq n} \sum_{l} t_{k l}}=0
$$


Proof. First we establish that 1) implies 2) and then we establish to converse.

We start by establishing 2.b. Suppose that 2.b fails and select $A \in I$ and $\tau>0$ such that

$$
J-\lim \frac{\sup _{k \geq n}\left\{\sum_{l} t_{k l}: l \in A\right\}}{\sup _{k \geq n} \sum_{l} t_{k l}} \neq 0
$$

and next select $\tau>0$ and a set $B \notin J$ such that $n \in B$ implies that

$$
\frac{\sup _{k \geq n}\left\{\sum_{k} t_{k l}: l \in A\right\}}{\sup _{k \geq n} \sum_{l} t_{k l}}>\frac{\tau}{2} .
$$

Let $r>4 / \tau$. Note that $B$ is infinite and define $x=\left(x_{j}\right)$ by $x_{j}=1$ if $j \notin A$ and $x_{j}=1+r$ for $j \in A$ and define the sequence $y=\left(y_{j}\right)$ by $y_{j}=1$ for all $j \in \mathbb{N}$. Note that $I-\lim \sup x=I-\lim \sup y=1$. Now observe that, for every $n \in B$, we have that

$$
\begin{aligned}
\sup _{k \geq n} \sum t_{k j} x_{j} & =\sup _{k \geq n}\left\{r \sum_{l} t_{k l} \chi_{A}(l)+\sum_{l} t_{k l}\right\} \\
& \geq r \sup _{k \geq n} \sum_{l} t_{k l} \chi_{A}(l)-\sup _{k \geq n} \sum_{l} t_{k l}
\end{aligned}
$$

and hence

$$
\begin{aligned}
\frac{\sup _{k \geq n}(T x)_{k}}{\sup _{k \geq n}(T y)_{k}} & \geq r \frac{\sup _{k \geq n} \sum_{l} t_{k l} \chi_{A}(l)}{\sup _{k \geq n} \sum_{k} t_{k l}}-\frac{\sup _{k \geq n} \sum_{l} t_{k l}}{\sup _{k \geq n} \sum_{l} t_{k l}} \\
& =r \frac{\tau}{2}-1>\frac{4}{\tau} \frac{\tau}{2}-1>1 .
\end{aligned}
$$

As $B \notin J$, it follows that

$$
\left\{n: \frac{\sup _{k \geq n}(T x)_{k}}{\sup _{k \geq n}(T y)_{k}} \geq 1\right\} \notin J
$$

$J-\lim \frac{\mu T x}{\mu T y} \neq 1$

Next we establish that 1 implies 2.a. Select $A \notin I$ and let $0<\varepsilon<1$ be given. Define the sequences $x=\left(x_{k}\right)$ and $y=\left(y_{k}\right)$ by $x_{k}=1$ if $k \in A$ and $x_{k}=\frac{\varepsilon}{2}$ for $k \notin A$ and $y_{k}=1$ for all $k \in \mathbb{N}$. Note that $I-\lim \sup x=$ $I-\lim \sup y=1$ and hence, by hypothesis, $J-\lim \frac{\mu T x}{\mu T y}=1$. Select $B \in I$ such that $n \notin B$ implies that

$$
1-\frac{\varepsilon}{2}<\frac{\sup _{k \geq n}\left\{\sum_{l} t_{k l} \chi_{A}(l)+\left(\frac{\varepsilon}{2}\right) \sum_{l} t_{k l} \chi_{A^{c}}(l)\right\}}{\sup _{k \geq n} \sum_{l} t_{k l}}<1+\frac{\varepsilon}{2} .
$$


Now observe that for $n \notin B$ it follows that

$$
\begin{aligned}
1-\frac{\varepsilon}{2} & <\frac{\sup _{k \geq n} \sum_{l} t_{k l} \chi_{A}(l)}{\sup _{k \geq n} \sum_{l} t_{k l}}+\left(\frac{\varepsilon}{2}\right) \frac{\sup _{k \geq n} \sum_{l} t_{k l} \chi_{A^{c}}(l)}{\sup _{k \geq n} \sum_{l} t_{k l}} \\
& \leq \frac{\sup _{k \geq n} \sum_{l} t_{k l} \chi_{A}(l)}{\sup _{k \geq n} \sum_{l} t_{k l}}+\frac{\varepsilon}{2}
\end{aligned}
$$

and hence

$$
1-\varepsilon<\frac{\sup _{k \geq n} \sum_{l} t_{k l} \chi_{A}(l)}{\sup _{k \geq n} \sum_{l} t_{k l}}
$$

Also observe that for $n \notin B$,

$$
\frac{\sup _{k \geq n} \sum_{l} t_{k l} \chi_{A}(l)-\left(\frac{\varepsilon}{2}\right) \sup _{k \geq n} \sum_{l} t_{k l} \chi_{A^{c}}(l)}{\sup _{k \geq n} \sum_{l} t_{k l}}<1+\frac{\varepsilon}{2}
$$

and hence

$$
\frac{\sup _{k \geq n} \sum_{l} t_{k l} \chi_{A}(l)}{\sup _{k \geq n} \sum_{l} t_{k l}}<1+\varepsilon .
$$

Combining our estimates now yields that

$$
\left\{n:\left|\frac{\sup _{k \geq n} \sum_{l} t_{k l} \chi_{A}(l)}{\sup _{k \geq n} \sum_{l} t_{k l}}-1\right|<\varepsilon\right\} \in F(J)
$$

and, as $\varepsilon>0$ was arbitrary,

$$
J-\lim \frac{\sup _{k \geq n}\left\{\sum_{l} t_{k l}: l \in A\right\}}{\sup _{k \geq n} \sum_{l} t_{k l}}=1 .
$$

We now show that conditions 2.a and 2.b are sufficient to yield condition 1. We start by making two observations which will by useful in our later computations. First, an argument similar to the one given in 1 implies 2.a yields that

$$
J-\lim \frac{\sup _{k \geq n} \sum_{l} t_{k l}}{\sup _{k \geq n}\left\{\sum_{l} t_{k l}: l \in F\right\}}=1
$$

whenever $F \notin I$. Secondly, we note that $A \in I$ and $F \notin I$ implies that

$$
J-\lim \frac{\sup _{k \geq n} \sum_{l}\left\{t_{k l}: l \in A\right\}}{\sup _{k \geq n}\left\{\sum_{l} t_{k l}: l \in F\right\}}=0 .
$$

The second observation can be established as follows. Let $\varepsilon>0$ be given and select $B \in F(J)$ such that $n \in B$ implies both that

$$
\sup _{k \geq n} \sum_{l}\left\{t_{k l}: l \in F\right\} \geq(1-\varepsilon) \sup _{k \geq n} \sum_{l} t_{k l}
$$

and that

$$
\frac{\sup _{k \geq n}\left\{\sum_{l} t_{k l}: l \in A\right\}}{\sup _{k \geq n} \sum_{l} t_{k l}}<\varepsilon .
$$


Note that $B$ can be found by intersecting two members of $F(J)$. Now, for $n \in B$, we have that

$$
0 \leq \frac{\sup _{k \geq n} \sum_{l}\left\{t_{k l}: l \in A\right\}}{\sup _{k \geq n}\left\{\sum_{l} t_{k l}: l \in F\right\}} \leq \frac{\sup _{k \geq n} \sum_{l}\left\{t_{k l}: l \in A\right\}}{(1-\varepsilon) \sup _{k \geq n} \sum_{l} t_{k l}}<\frac{\varepsilon}{1-\varepsilon}
$$

and, as $\varepsilon>0$ was arbitrary, the second claim follows.

We can now conclude the proof of the result. Let $x, y$ be bounded sequences in $P_{\delta}$ and suppose that $I-\lim \sup x=I-\lim \sup y=\beta$. Note that $\beta \geq \delta$ and let $0<\varepsilon<\beta$. Now select sets $A \in I, E \notin I$ and $F \notin I$ such that

(1) $x_{l}$ and $y_{l}$ are less than $\beta+\varepsilon$ for all $l \notin A$, and

(2) $y_{l}$ is greater than $\beta-\varepsilon$ for all $l \in F$.

Now observe that

$$
\sup _{k \geq n} \sum_{l} t_{k l} x_{l} \leq(\beta+\varepsilon) \sup _{k \geq n} \sum_{l}\left\{t_{k l}: l \notin A\right\}+\|x\| \sup _{k \geq n} \sum_{l}\left\{t_{k l}: l \in A\right\}
$$

and that

$$
\sup _{k \geq n} \sum_{l} t_{k l} y_{l} \geq \sup _{k \geq n} \sum_{l}\left\{t_{k l} y_{l}: l \in F\right\}>(\beta-\varepsilon) \sup _{k \geq n} \sum_{l}\left\{t_{k l}: l \in F\right\} .
$$

and hence

$$
\begin{aligned}
\frac{\sup _{k \geq n} \sum_{l} t_{k l} x_{l}}{\sup _{k \geq n} \sum_{l} t_{k l} y_{l}} & \leq \frac{\|x\| \sup _{k \geq n} \sum_{l}\left\{t_{k l}: l \in A\right\}}{(\beta-\varepsilon) \sup _{k \geq n} \sum_{l}\left\{t_{k l}: l \in F\right\}} \\
& +\frac{(\beta+\varepsilon) \sup _{k \geq n} \sum_{l}\left\{t_{k l}: l \notin A\right\}}{(\beta-\varepsilon) \sup _{k \geq n} \sum_{l}\left\{t_{k l}: l \in F\right\}}
\end{aligned}
$$

Let $\nu>0$ and select $B_{1}, B_{2} \in F(J)$ such that $n \in B_{1}$ implies

$$
\frac{\sup _{k \geq n} \sum_{l}\left\{t_{k l}: l \in A\right\}}{\sup _{k \geq n}\left\{\sum_{l} t_{k l}: l \in F\right\}}<\nu
$$

and $n \in B_{2}$ implies that

$$
\frac{\sup _{k \geq n} \sum_{l} t_{k l}}{\sup _{k \geq n}\left\{\sum_{l} t_{k l}: l \in F\right\}}<1+\nu .
$$

Now, if $n \in B_{1} \cap B_{2}$, then

$$
\frac{\sup _{k \geq n} \sum_{l} t_{k l} x_{l}}{\sup _{k \geq n} \sum_{l} t_{k l} y_{l}}<\frac{\nu}{(\beta-\varepsilon)}\|x\|+\frac{\beta+\varepsilon}{\beta-\varepsilon}(1+\nu)
$$

Observe that for any $\delta>0$ we can select positive values of $\varepsilon$ and $\nu$ such that

$$
1<\frac{\nu}{(\beta-\varepsilon)}\|x\|+\frac{\beta+\varepsilon}{\beta-\varepsilon}(1+\nu)<1+\delta
$$


Now observe that a similar argument yields there a set $B_{3} \in F(J)$ such that $n \in B_{3}$ implies that

$$
\frac{\sup _{k \geq n} \sum_{l} t_{k l} y_{l}}{\sup _{k \geq n} \sum_{l} t_{k l} x_{l}}<1+\delta
$$

and hence

$$
1-\delta<\frac{1}{1+\delta}<\frac{\sup _{k \geq n} \sum_{l} t_{k l} x_{l}}{\sup _{k \geq n} \sum_{l} t_{k l} y_{l}}
$$

Now note that $B=B_{1} \cap B_{2} \cap B_{3} \in F(J)$ and that

$$
1-\delta<\frac{\sup _{k \geq n} \sum_{l} t_{k l} x_{l}}{\sup _{k \geq n} \sum_{l} t_{k l} y_{l}}<1+\delta
$$

for all $n \in B$, thus establishing the result.

\section{REFERENCES}

[1] J. Connor, J. A. Fridy and C. Orhan, Core equality results for sequences, J. Math. Anal. Appl., 321 (2) (2006), 515-523.

[2] Jeff Connor, Two valued measures and summability, Analysis, 10 (4) (1990), 373-385.

[3] _ A Topological and Functional Analytic Approach to Statistical Convergence, Analysis of divergence (Orono, ME, 1997), Appl. Numer. Harmon. Anal., Birkhäuser Boston, Boston, MA, 1999, pp. 403-413.

[4] Kamil Demirci, I-limit superior and limit inferior, Math. Commun., 6 (2) (2001), 165-172.

[5] H. Fast, Sur la convergence statistique, Colloquium Math., 2 (1951), 241-244 (1952).

[6] J. A. Fridy, Minimal rates of summability, Canad. J. Math., 30 (4) (1978), 808-816.

[7] _ On statistical convergence, Analysis, 5 (4) (1985), 301-313.

[8] _ Statistical limit points, Proc. Amer. Math. Soc., 118 (4) (1993), 1187-1192.

[9] J. A. Fridy and C. Orhan, Statistical core theorems, J. Math. Anal. Appl., 208 (2) (1997), 520-527.

[10] Hafize Gumus, Jeff Connor and Fatih Nuray, Asymptotic i-equivalence of two number sequences and asymptotic i-regular matrices, Chin. J. Math. 2014, (2014), no. Article ID 805857, 5 .

[11] Pavel Kostyrko, Tibor Šalát and Władysław Wilczyński, I-convergence, Real Anal. Exchange, 26 (2) (2000/01), 669-685.

[12] Jinlu Li, Asymptotic equivalence of sequences and summability, Internat. J. Math. Math. Sci., 20 (4) (1997), 749-757.

[13] Mousa S. Marouf, Asymptotic equivalence and summability, Internat. J. Math. Math. Sci., 16 (4) (1993), 755-762.

[14] F. Patterson and E. Savaş, On asymptotically lacunary statistical equivalent sequences, Thai J. Math., 4 (2) (2006), 267-272.

[15] Richard F. Patterson, On asymptotically statistical equivalent sequences, Demonstratio Math., 36 (1) (2003), 149-153.

[16] I. P. Pobyvanets, Asymptotic equivalence of some linear transformations, defined by a nonnegative matrix and reduced to generalized equivalence in the sense of Cesàro and Abel, Mat. Fiz. (1980), no. 28, 83-87, 123. 
(Received: March 4, 2015)

\author{
Jeff Connor \\ Department of Mathematics \\ Ohio University \\ Athens, Ohio \\ U.S.A. \\ connorj@ohio.edu \\ Hafize Gumus \\ Faculty of Eregli Education \\ Necmettin Erbakan University \\ Eregli, Konya \\ Turkey
}

